\title{
The Effect of Cucurbit[n]uril on the Solubility, Morphology, and the Photophysical Properties of Nonionic Conjugated Polymers in an Aqueous Medium
}

\author{
DÖNÜS TUNCEL, MÜGE ARTAR, SALTUK BUGRA HANAY \\ Department of Chemistry and Institute of Materials Science, Institution and Nanotechnology, Bilkent University, Bilkent, \\ Ankara 06800, Turkey
}

Received 14 June 2010; accepted 4 August 2010

DOI: $10.1002 /$ pola.24284

Published online 20 September 2010 in Wiley Online Library (wileyonlinelibrary.com).

\begin{abstract}
The effects of cucurbit[ $n]$ uril on the dissolution and the photophysical properties of nonionic conjugated polymers in water are described. For this purpose, a fluorine-based polymer, namely, poly[9,9-bis $\{6(N, N$-dimethylamino $)$ hexyl\}fluoreneco-2,5-thienylene (PFT) was synthesized and characterized by spectroscopic techniques including 1D and 2D NMR, UV-vis, fluorescent spectroscopy, and matrix-assisted laser desorption mass spectrometry (MALDI-MS). For the first time, it was demonstrated that a nonionic conjugated polymer can be made soluble in water through an inclusion complex formation with CB8. The structure of the complex was elucidated by NMR experiments including ${ }^{1} \mathrm{H}$ and selective 1D-NOESY. This complex emits green and is highly fluorescent with fluorescent quantum yield of $35 \%$. In contrast, CB6 or water-soluble CB7
\end{abstract}

although they are chemically identical to CB8 do not have any effect on the dissolution and photophysical properties of PFT. By preparing a protonated version of $\mathrm{PFT}$, the optical properties of PFT in methanol, protonated PFT and PFT@CB8 in water have been studied and compared. It was also observed that the morphology of the polymer PFT was affected by the presence of CB8. Thus CB8-assisted self-assembly of polymer chains leads to vesicles formation; these structures were characterized by DLS, AFM, SEM, and TEM fluorescent optical microscopy. (c) 2010 Wiley Periodicals, Inc. J Polym Sci Part A: Polym Chem 48: 4894-4899, 2010

KEYWORDS: conjugated polymers; cucurbituril; self-assembly; supramolecular structures; water-soluble polymers
INTRODUCTION Conjugated polymers are of great interest because of their broad applications including in the construction of optoelectronic devices, chemical and biological sensors. ${ }^{1-8}$ Among many other conjugated polymers, fluorinebased polymers have been extensively studied owing to their high photoluminescent quantum yields and chemical stability; they can also be modified easily without disrupting their aromatic backbones to tune their solubility and optical properties. ${ }^{9,10}$ Water-soluble conjugated polymers are particularly appealing because they can be used in biological applications and in the construction of optoelectronic devices in non-noxious solvent. ${ }^{4,5,8}$ Water-soluble conjugated polymers are usually obtained by attaching ionic or hydrophilic side chains to polymer backbone. ${ }^{11-14}$ However, the hydrophobic aromatic backbone of conjugated polymers often cause an intrinsic aggregation, which leads to poor water-solubility and low fluorescence quantum yields that are not desirable for many applications. Thus, it is highly sought-after to develop strategies to prevent or decrease the polymer intrinsic aggregation and improve water-solubility as well as to increase their fluorescent quantum yields. For this purpose, many approaches have been adopted that include the incorpora- tion of bulky groups, ${ }^{14}$ interaction with surfactants, ${ }^{15,16}$ and encapsulating the backbone of the conjugated polymers with a suitable macrocyle. ${ }^{17-19}$ It was shown that the encapsulation of conjugated polymers by cyclodextrin derivatives could indeed prevent $\pi-\pi$ interaction between the polymer chains, which in turn provides an enhanced environmental stability and increased photo and electroluminescence efficiencies. $^{17-19}$

Cucurbit $[n]$ uril ( $\mathrm{CB}[n], n=5-10$ ), a family of macrocycles synthesized from acid catalyzed condensation of glycoluril and formaldehyde. ${ }^{20-22}$ They possess a hydrophobic, nonpolarizable cavity, and hydrophilic portals decorated with carbonyl groups. They have been used in the synthesis of a wide variety of supramolecular assemblies including molecular machines and switches. ${ }^{23-28}$ Among them, CB5 and CB7 are water-soluble; however, CB5 has a quite small cavity to encapsulate guests. The effect of $\mathrm{CB}$ encapsulation on the photophysical and chemical properties of dyes have been investigated in some details. ${ }^{29-32}$ However, these efforts were more focused on encapsulation of small dyes by watersoluble CB7. There are only a few examples in the use of CB7 for encapsulating conjugated polymers. ${ }^{33-35}$ However, to 


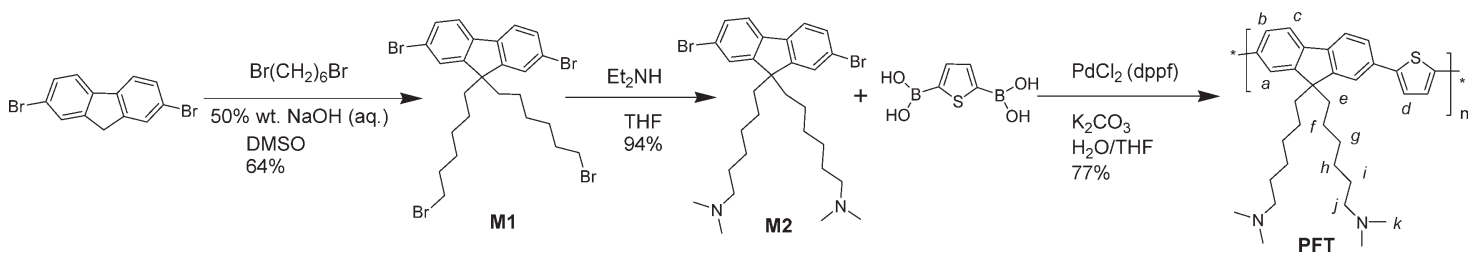

SCHEME 1 Synthetic scheme for poly[9,9-bis $\{6(N, N$-dimethylamino)hexyl $\}$ fluorine-co-2,5-thienylene (PFT).

the best of our knowledge, there is no report on the CBdriven dissolution and photoluminescent quantum enhancement of nonionic conjugated polymers in water.

In this article, the effect of $\mathrm{CB}[n]$ on the dissolution and photophysical properties of nonionic conjugated polymers in water are described. For this purpose, a fluorine-based polymer, namely, poly[9,9-bis $\{6(N, N$-dimethylamino $)$ hexyl $\}$ fluorene-co-2,5-thienylene (PFT) was synthesized and characterized by spectroscopic techniques including 1D and 2D NMR, UV-vis, fluorescent and matrix-assisted laser desorption mass spectrometry (MALDI-MS). For the first time, it was demonstrated that a nonionic conjugated polymer can be made soluble in water by forming an inclusion complex with CB8. The structure of the complex was elucidated by NMR experiments including ${ }^{1} \mathrm{H}$ and selective 1D-NOESY. This inclusion complex emits green and is highly fluorescent with fluorescent quantum yield of $35 \%$. In contrast, CB6 or water-soluble CB7 although they are chemically identical to CB8 do not have any effect on the dissolution and photophysical properties of PFT. Moreover, a protonated PFT has also been prepared for comparison. Thus, optical properties of PFT in methanol, protonated PFT and PFT@CB8 in water have been studied and compared. It was also observed that the morphology of the polymer PFT was affected by the presence of CB8. Thus, CB8assisted self-assembly of polymer chains leads to vesicles formation; these structures were characterized by DLS, AFM, SEM, TEM, and fluorescent optical microscopy.

\section{RESULTS AND DISCUSSION}

Poly[9,9-bis $\{6$-( $N, N$-dimethylamino $)$ hexyl $\}$ fluorine- $\mathrm{Co}$-2,5-thienylene (PFT) was synthesized according to reaction Scheme 1 in good yield. After the synthesis of monomer M1 following the literature preparation, ${ }^{7}$ it was converted into M2 by treating excess dimethylamine solution in THF. PFT was synthesized by Pd-catalyzed Suzuki coupling of M2 with 2,5-thiophenediboronic acid.

PFT is soluble in a wide range of solvents such as THF, $\mathrm{CHCl}_{3}$, methanol, and aqueous acid. Its structure was confirmed by ${ }^{1} \mathrm{H}, 2 \mathrm{D}$-COSY, and ${ }^{13} \mathrm{C}$-NMR. Figure 1 (a) shows the ${ }^{1} \mathrm{H}-\mathrm{NMR}$ spectrum of PFT in $\mathrm{CDCl}_{3}$. It was also characterized by FTIR spectroscopy (Supporting Information Fig. S10).

Although we have tried various conditions and solvents for molecular weight determination of PFT using gel permeation chromatography (GPC), we were not able to obtain good results probably due to the irreversible adsorption of the polymer onto the column packing material. However, MALDIMS provided satisfactory results. Supporting Information Fig- ure S1 depicts its MALDI-MS spectrum. Each line separated by a mass of $\sim 500 \mathrm{Da}$ which corresponds to the mass of one repeat unit. The spectrum shows up to 15 repeat units of PFT.

PFT is a nonionic conjugated polymer that its side chains are terminated with tertiary amine groups; this allows PFT to be soluble in methanol but not in water. First, to find out whether CB homologues could assist the dissolution of PFT in water, initially water soluble CB7 was tried. To this end, solid PFT (0.02 mmol, per repeat unit) was suspended in water $(10 \mathrm{~mL})$, sonicated for $10 \mathrm{~min}$, and then two equivalent of CB7 per polymer repeat unit were added. The resulting suspension was gently heated and sonicated, however, even after a week only slight color change was observed. A similar result was obtained when the same procedure was repeated for CB6. Neither water soluble CB7, nor nonwater soluble CB6 aided the dissolution of PFT. On the other hand, under the same conditions nonwater soluble CB8 was able to solubilize PFT by producing a bright green emitting aqueous solution.

To gain further insight on the origin of dissolution process and elucidate the structure of PFT@CB8, NMR experiments were performed. Since nonprotonated PFT does not dissolve in water (even sparingly) and the ${ }^{1} \mathrm{H}$-NMR spectrum of the protonated PFT shows very broad peaks (Supporting Information Fig. S2) that make the analysis difficult, we had to compare the spectra of PFT in the absence and the presence of CB8 in $\mathrm{CDCl}_{3}$ and $\mathrm{D}_{2} \mathrm{O}$, respectively. Figure $1(\mathrm{~b})$ shows the ${ }^{1} \mathrm{H}$-NMR spectrum of PFT in the presence of CB8 in $\mathrm{D}_{2} \mathrm{O}$. As

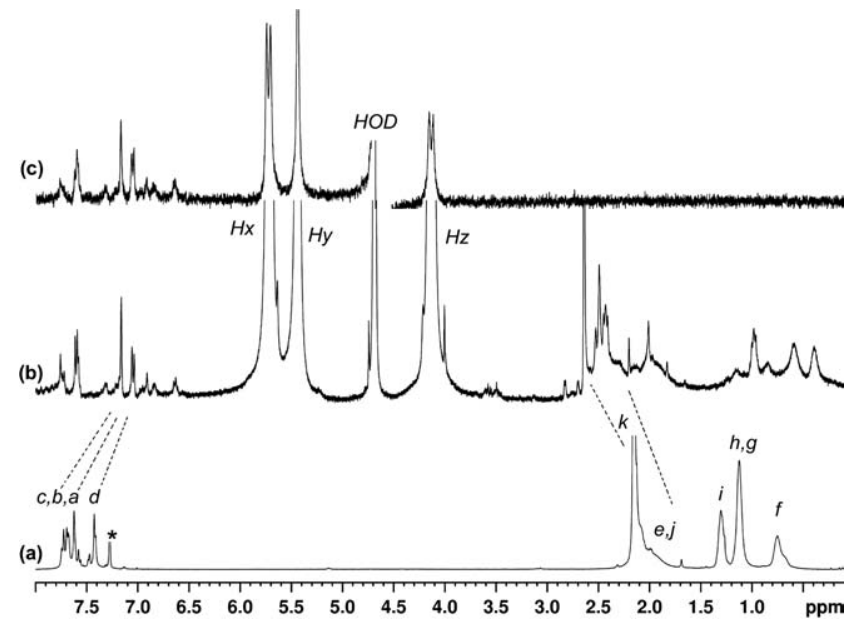

FIGURE $1{ }^{1} \mathrm{H}-\mathrm{NMR}(400 \mathrm{MHz}, 298 \mathrm{~K})$ spectra of (a) PFT in $\mathrm{CDCl}_{3}$, (b) PFT@CB8 (3 mM) in $\mathrm{D}_{2} \mathrm{O}$, and (c) 1D-NOESY spectrum of PFT@CB8 in $\mathrm{D}_{2} \mathrm{O}$, proton $\mathrm{Hx}$ at 5,73 ppm was irradiated, mixing time D: $100 \mathrm{~ms}\left({ }^{*}\right.$ for $\mathrm{CHCl}_{3}$ ). 


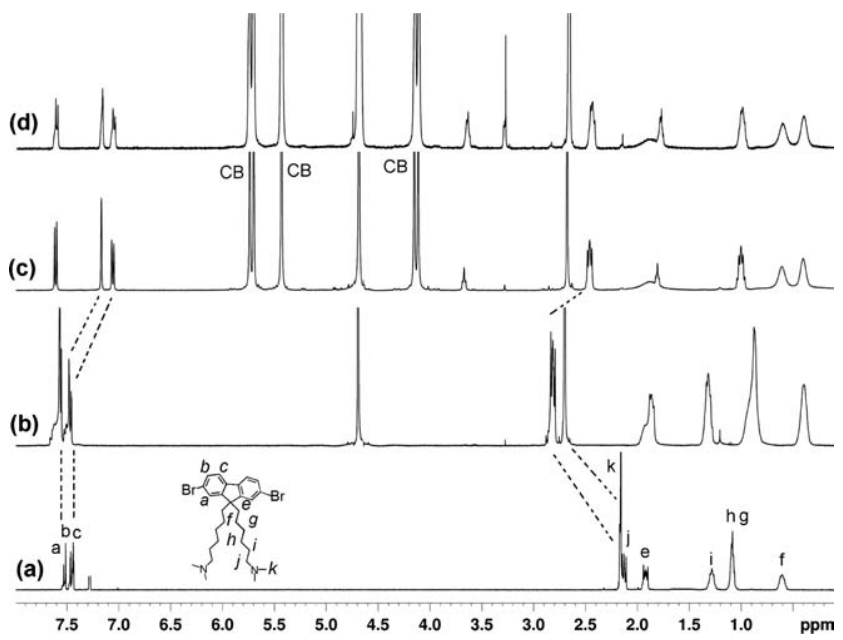

FIGURE $2{ }^{1} \mathrm{H}-\mathrm{NMR}\left(400 \mathrm{MHz}, 298 \mathrm{~K}\right.$ ) spectra of (a) $\mathrm{M} 2$ in $\mathrm{CDCl}_{3}$, (b) protonated $\mathrm{M} 2(\mathrm{M} 2 \mathrm{H})$ in $\mathrm{D}_{2} \mathrm{O}$, (c) $\mathrm{M} 2 \mathrm{H}(4 \mathrm{mM})$ in the presence of 1 equiv $\mathrm{CB} 8$ in $\mathrm{D}_{2} \mathrm{O}$, and (d) $\mathrm{M} 2(4 \mathrm{mM})$ in the presence of 1 equiv $\mathrm{CB} 8$ in $\mathrm{D}_{2} \mathrm{O}$.

can be seen, there are significant changes in the spectrum 1b compared to the spectrum 1a. The peaks for the methyl protons attached to nitrogen atoms and the methylene groups next to nitrogen shifted $0.6 \mathrm{ppm}$ downfield. Significant changes were also observed in the aromatic region. The peaks for the fluorene protons shifted about $0.5-1.0 \mathrm{ppm}$ upfield. Here one might question that whether these changes in the spectrum of PFT@CB8 could be attributed to the solvent effect because these two spectra were recorded in two solvents with different polarities.

To clarify this possibility and understand better the interaction of CB8 with PFT, we have carried out some control experiments in which ${ }^{1} \mathrm{H}-\mathrm{NMR}$ spectra of protonated M2 (M2H) in the absence and in the presence of CB8 and ${ }^{1} \mathrm{H}-\mathrm{NMR}$ spectrum of nonprotonated M2 in the presence of CB8 were recorded in the same solvent $\left(D_{2} O\right)$ (Fig. 2). We also found out that M2 before protonation was not soluble in water but it became soluble in the presence of CB8. The interactions between CB8 and M2 have been further investigated by 2D-COSY and 1DNOESY experiments. Figure $2(b, c)$ show the ${ }^{1} \mathrm{H}-\mathrm{NMR}$ spectra of $\mathrm{M} 2 \mathrm{H}$ in the absence and in the presence of CB8 in $\mathrm{D}_{2} \mathrm{O}$, respectively. As can be seen from the spectrum $2 c$ in the presence of CB8, the peaks of fluorene protons $a$ and $b$ shifted about 0.6 ppm upfield whereas the peak for proton $c$ shifted $0.1 \mathrm{ppm}$ downfield. There are also some changes in the peaks of side chain protons. For instance, the peak for proton $j$ shifted 0.3 ppm upfield in the presence of CB8. To understand the nature of the interaction, we performed selective 1D-NOESY experiments. For this, selectively all well-resolved peaks were irradiated using varying mixing times. 1D-NOESY NMR shows that all correlation peaks are negative. When the peaks of fluorene protons $(a, b$, and $c)$ were irradiated, large negative NOE were detected for the peaks of CB8 $(H x$ and $H y)$ at 5.71 and 5.44 ppm, respectively. The irradiation of peaks for $\mathrm{Hx}$ and $\mathrm{Hy}$ at 5.71 and 5.44, respectively, show large negative NOEs for fluorene protons $a, b$, and $c$; but no correlations were observed between side chain aliphatic protons and CB protons $H x$ and $\mathrm{Hy}$. However, the irradiation of peak for $\mathrm{Hz}$ of CB8 at $4.12 \mathrm{ppm}$ shows negative strong NOE on the methyl protons $(k)$ of aliphatic side chains (see Supporting Information Fig. S6 for selective 1D-NOESY experiments). The observation of NOE correlations between $H x$ and $H y$ protons of CB8 and protons $a, b$, and $c$ of fluorene and about $0.6 \mathrm{ppm}$ upfield shift in the peaks of protons $b$ and $c$ of fluorene in ${ }^{1} \mathrm{H}$-NMR spectrum indicate that the aromatic protons were encapsulated by CB8. Moreover, ${ }^{1} \mathrm{H}$ and 1D-NOESY experiments suggest that the methyl groups are in close proximity with $\mathrm{Hz}$ protons of CB8 and the nitrogens of the dimethyl amine groups are CB-assisted protonated because the ${ }^{1} \mathrm{H}-\mathrm{NMR}$ spectra of protonated M2@CB8 [Fig. 2(c)], and nonprotonated M2@CB8 are identical [Fig. 2(d)]. It is known that especially CB7 upon complexation with fluorescent dyes could cause a $\mathrm{p} K_{\mathrm{a}}$ shift in protonation. ${ }^{29,30}$

From the control experiments, it is clear that the changes in the ${ }^{1} \mathrm{H}$-NMR spectrum of PFT@CB8 are not due to the solvent effect but they are indeed due to the CB8 effect on the PFT. We have also performed selective 1D-NOESY experiments on the sample of PFT@CB8 by irradiating selectively the peaks of CB8 at 5.73, 5.44, and 4.11 (Supporting Information Fig. S7). The results are very similar to $1 \mathrm{D}$-NOESY NMR experiments performed for the complex M2H@CB8 and again all correlation peaks are negative. The correlations between $H x$ and $H y$ protons of CB8 and aromatic protons were observed. Figure 1(c) displays the 1D-NOESY spectrum of PFT@CB8 in which the proton $H x$ at 5.73 ppm was irradiated. The peak of CB8 protons $\mathrm{Hz}$ and protons of methyl groups of side chain are also correlated. These results clearly indicate that the some part of the aromatic backbone of the PFT was encapsulated by CB8, and the methyl groups of the side chains are in a close proximity with CB8 as suggested in Figure 3. Probably, again similar to M2@CB8 complex the nitrogens of dimethyl groups are protonated by coordinating to the carbonyl groups of CB8.

Second, to explore the effect of CB8 on the optical properties of PFT, we have compared the absorption and emission spectra of PFT in $\mathrm{MeOH}$, protonated PFT in water, and PFT@CB8 inclusion complex in water. Figure 4 (a) illustrates their absorbance spectra. PFT in methanol absorbs at $436 \mathrm{~nm}$ with a strong shoulder at $457 \mathrm{~nm}$, while the spectra of protonated

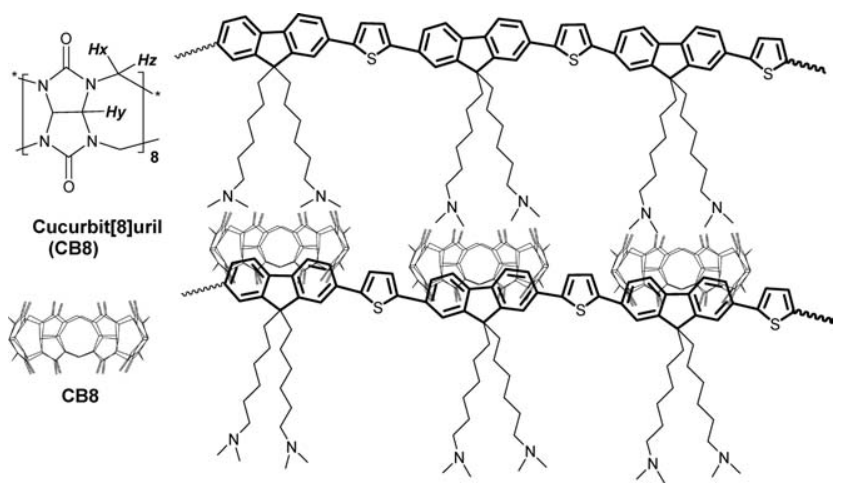

FIGURE 3 Suggested structure based on the ${ }^{1} \mathrm{H}$ and 1D-NOESY NMR data for the PFT@CB8 complex formation. 

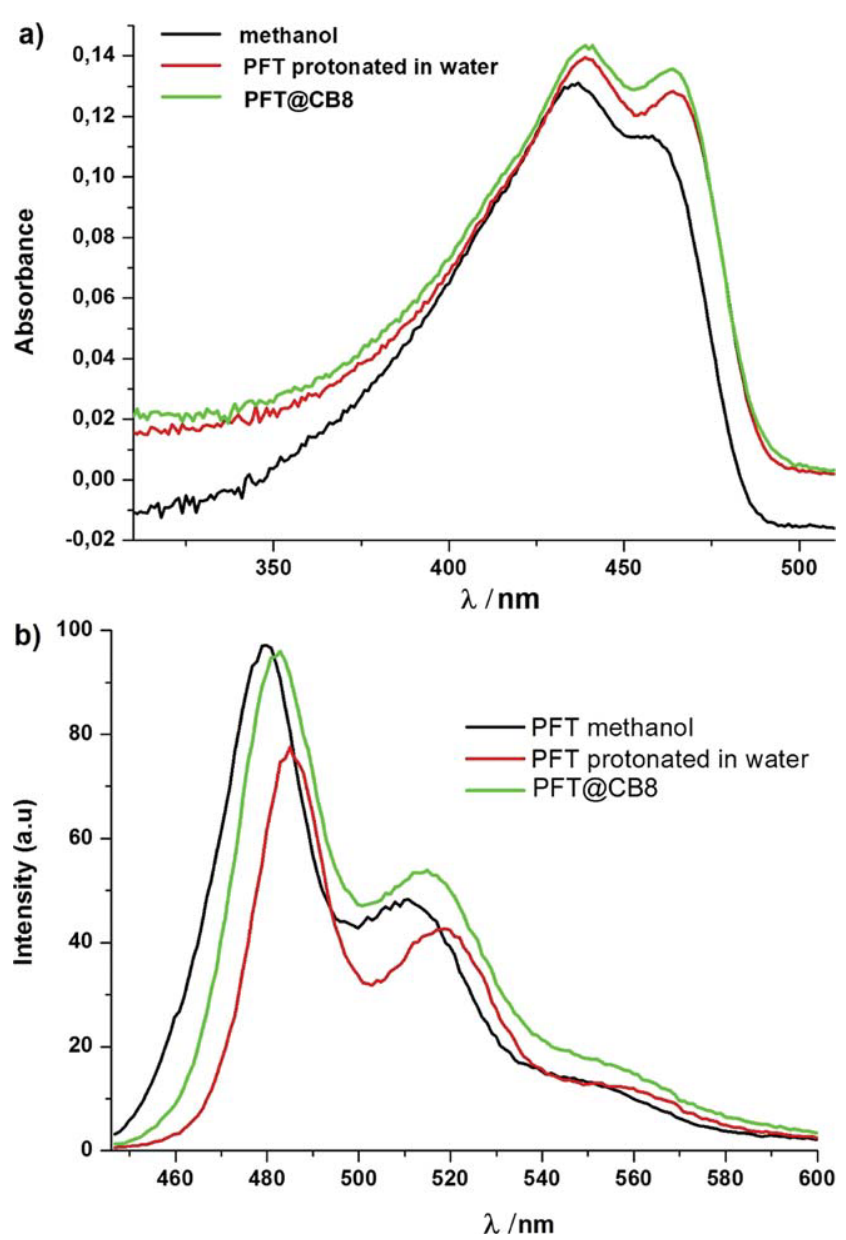

FIGURE 4 (a) UV-vis absorption, (b) emission spectra of PFT in methanol, protonated PFT in water and PFT@CB8 in water.

PFT in water and PFT@CB8 which are almost identical exhibit two absorption maxima at 439 and $463 \mathrm{~nm}$ that are 3 and 6 $\mathrm{nm}$, respectively, red-shifted relative to PFT in methanol.

When PFT in methanol is excited at $436 \mathrm{~nm}$, an emission maximum at $479 \mathrm{~nm}$ with two vibronic peaks at 511, 549 are observed, whereas the protonated PFT emits at $485 \mathrm{~nm}$ with two vibronic bands at 519 and $558 \mathrm{~nm}$. The emission spectrum of PFT@CB8 reveals a peak at 483 nm with two vibronic bands at $515,552 \mathrm{~nm}$ which is $2 \mathrm{~nm}$ blue shifted relative to the protonated PFT and $4 \mathrm{~nm}$ red shifted relative to PFT in methanol [Fig. 4(b)]. Although the differences in the emission wavelength of these three samples are not significant, there are substantial differences in their fluorescent quantum yields which are $45 \%, 35 \%$, and $8 \%$ for PFT in methanol, PFT@CB8 in water, and protonated PFT in water, respectively. Table 1 summarizes the photophysical parameters of PFT in methanol, protonated PFT, and PFT@CB8.

To understand further the origin of emission enhancement in PFT caused by the presence of CB8, dynamic light scattering (DLS) measurements were conducted. DLS of the solution of PFT@CB8 in water indicated the presence of aggregates with an average diameter of $700 \mathrm{~nm}$. After filtration the solution using $0.45 \mu \mathrm{m}$ syringe filter, the particle size decreased to $200 \mathrm{~nm}$ (Supporting Information Fig. S5).

These aggregates were also examined by atomic force microscopy (AFM), scanning electron microscopy (SEM), transmission electron microscopy (TEM), and fluorescent optical microscopy (FOM). AFM shows spherical particles with diameter ranging from $50 \mathrm{~nm}$ to $1 \mu \mathrm{m}$ [Fig. 5(a)]. When the SEM images of these particles were taken (Supporting Information Fig. S8), it was observed that the particles look like collapsed and deflated vesicles. To confirm whether these particles have vesicle like structures, we further examined them by TEM. The TEM micrographs confirm the formation of vesicles [Fig. 5(b), and Supporting Information Fig. S9]. These particles were also characterized by FOM; the fluorescence micrograph shows the presence of green emitting spherical particles [Fig. $5(c)]$. These results suggest that in the presence of CB8, PFT self-assemble to form vesicles. CB-triggered vesicles formation was also demonstrated by Kim and coworkers ${ }^{36}$ in a charge-transfer ternary complex. Currently, it is not clear to us how these vesicles form; however, we think the following structure is the most plausible one according to the experimental evidences. In this proposed structure, the hydrophobic alkyl chains are joined by CB8s through dimethylamine parts; while one of the portals of CB8s interacting with aromatic part of the PFT, the other portal is exposed to water molecules by causing vesicles formation (Fig. 6). (a)

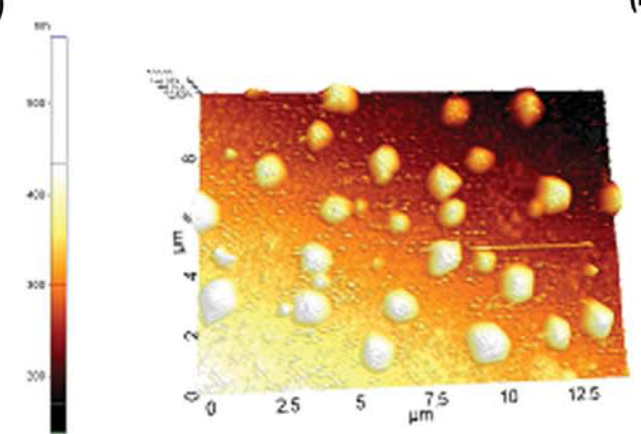

(b)

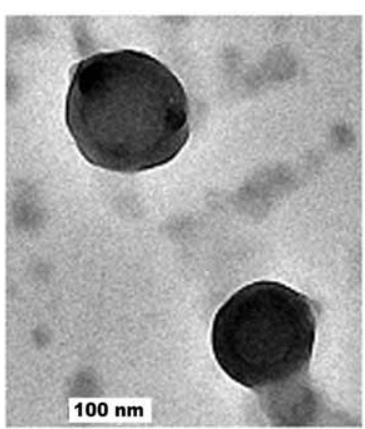

(c)

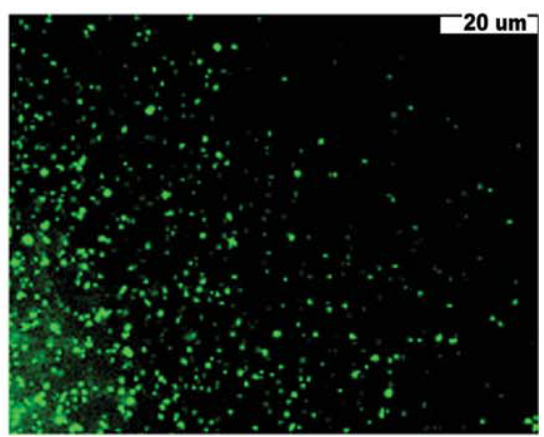

FIGURE 5 (a) AFM, (b) TEM, and (c) FOM (40× magnification) micrographs of nanoparticles formed by self-assembly PFT in the presence of CB8 (concentration of PFT(per repeat unit): $0.08 \mathrm{mM})$. 
TABLE 1 Photophysical Data for PFT in Methanol, Protonated PFT, and PFT@CB8

\begin{tabular}{lllll}
\hline & $\lambda_{\max }$ abs $(\mathrm{nm})$ & $\lambda_{\max } \mathrm{em}(\mathrm{nm})$ & $\Phi_{\mathrm{f}}{ }^{\mathrm{a}}(\%)$ & $\varepsilon_{\max }{ }^{\mathrm{b}}\left(10^{4} \mathrm{M} / \mathrm{cm}\right)$ \\
PFT in $\mathrm{MeOH}$ & $436,457(\mathrm{sh})$ & $479,511,549$ & 45 & 4.2 \\
Protonated PFT $^{\mathrm{c}}$ & 439,464 & $485,519,558$ & 8 & 2.2 \\
PFT@ CB8 & 439,463 & $483,515,552$ & 35 & 2.1
\end{tabular}

a Quantum yields were measured relative to quinine sulfate $0.1 \mathrm{M} \mathrm{H}_{2} \mathrm{SO}_{4}\left(\Phi_{\mathrm{f}}=55 \%\right)$.

${ }^{b}$ Per repeat unit.

${ }^{\mathrm{c}}$ Spectra were recorded in water.

\section{EXPERIMENTAL}

CB6, CB7, and CB8 were prepared according to literature. ${ }^{20}$ All other reagents and solvents were of the commercial reagent grade and used without further purification except where noted. Nuclear magnetic resonance (NMR) spectra were recorded on Bruker Avance DPX-400 MHz spectrometer. The samples were dissolved in $\mathrm{D}_{2} \mathrm{O}$ with DSS (3-(trimethylsilyl)-1-propanesulfonic acid sodium salt) as an external standard. 1D-NOESY NMR experiments are performed using selnogp by varying the mixing time (D) at $25^{\circ} \mathrm{C}$. Mass spectra were taken on Applied Biosystems MALDI-TOF MS mass spectrometer equipped with a YAG-Laser $(\lambda=335 \mathrm{~nm})$ at $10^{-7}$ Torr and delayed extraction mode (delay $300 \mathrm{~ns}$ ) by changing the laser intensity, if necessary. All spectra were acquired in reflector mode using $20 \mathrm{kV}$ acceleration potential, and they were the average of 60 laser shots. Then, data were transferred to a personal computer for further processing. 2,4,6Trihydroxyacetophenone (THAP) was chosen as the MALDI matrix. SEM images were taken from a Quanta 200 FEG electron microscope. TEM experiments were performed with a FEI (model Tecnai G2F30) accelerating voltage of $200 \mathrm{kV}$. The samples were prepared by dropping the solution of PFT@CB8 in water on a copper gird coated with a carbon membrane. AFM images were taken from XE-100E, PSIA model microscope, and samples were prepared by drop casting the solution of PFT@CB8 on a silicon wafer. DLS measurements were conducted using a Malvern NanoS spectrometer at a wavelength of $633 \mathrm{~nm}$ by using laser as the light source; light intensity was measured at an angle of $173^{\circ}$.

Synthesis of \{6-[2,7-Dibromo-9-(6-dimethylamino-hexyl)9H-fluoren-9-yl]-hexyl\}-dimethyl-amine (M2)

2,7-Dibromo-9,9-bis(6-bromo-hexyl)-9H-fluorene (M1) (1.00 g, $1.54 \mathrm{mmol}$ ) dissolved in dry THF (5 mL) and flushed with argon. The solution was cooled down to $-78{ }^{\circ} \mathrm{C}$. $2 \mathrm{M}$ solution of dimethylamine in THF (10 mL, $20 \mathrm{mmol})$ was added under argon. The mixture was brought to rt and stirred for $24 \mathrm{~h}$ at rt. THF was removed under reduced pressure, and $0.1 \mathrm{M} \mathrm{NaOH}(5 \mathrm{~mL})$ and $\mathrm{CHCl}_{3}(10 \mathrm{~mL})$ were added. The mixture was stirred for $30 \mathrm{~min}$, and the organic layer was separated, dried over $\mathrm{MgSO}_{4}$. The solvent was removed under reduced pressure. Purification by flash chromatography on silica gel (ethylacetate/MeOH/ $\mathrm{Et}_{3} \mathrm{~N}, 88: 10: 2$ ) afforded M2 as a white waxy solid $(0.85 \mathrm{~g}, 94 \%) .{ }^{1} \mathrm{H}-\mathrm{NMR}(400 \mathrm{MHz}$, $\left.\mathrm{CDCl}_{3}, \delta\right): 0.62(\mathrm{~m}, 2 \mathrm{H}), 1.08(\mathrm{~m}, 4 \mathrm{H}), 1.26(\mathrm{~m}, 2 \mathrm{H}), 1.95(\mathrm{~m}$, 2H), $1.09(\mathrm{~m}, 8 \mathrm{H}), 7.47-7.59(\mathrm{~m}, 3 \mathrm{H}) ;{ }^{13} \mathrm{C}-\mathrm{NMR}(100 \mathrm{MHz}$, $\mathrm{CDCl}_{3}, \delta$ ): 145.12, 135.43, 132.21, 130.23, 127.80, 122.50,
56.67, 43.43, 42.90, 41.43, 30.60, 29.32, 28.10, 24.12; Elemental analysis: calcd for $\mathrm{C}_{29} \mathrm{H}_{42} \mathrm{Br}_{2} \mathrm{~N}_{2}$ : C 60.21, $\mathrm{H} 7.32, \mathrm{~N}$ 4.84; found: C 60.05, H 7.23, N 4.75.

Poly[9,9-bis $\{6$ - $(N, N$-dimethylamino $)$ hexyl $\}$ fluorene-co-2,5-thienylene (PFT): M2 (400 mg, $0.691 \mathrm{mmol}$ ), 2,5-thiophenediboronic acid (119 $\mathrm{mg}, 0.692 \mathrm{mmol}$ ), $\mathrm{PdCl}_{2}(\mathrm{dppf})$ (dppf = diphenylphosphinoferrocene, $10 \mathrm{mg}$ ), and potassium carbonate $(500 \mathrm{mg}, 3.61 \mathrm{mmol})$ were placed in a $25-\mathrm{mL}$ round-bottom flask. A mixture of water $(3 \mathrm{~mL})$ and THF $(5 \mathrm{~mL})$ was added to the flask and the reaction vessel was degassed. The mixture was stirred at $80{ }^{\circ} \mathrm{C}$ for $24 \mathrm{~h}$ under argon, and then precipitated into water. The polymer was filtered and washed with water, redissolved in THF, and precipitated into water. The precipitate was filtered and then dried under vacuum for $24 \mathrm{~h}$ to afford PFT (400 mg, 77\%) as green-brown-

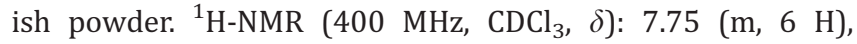
7.52 (m, 2 H), 2.25 (br, 16 H), 1.45 (br, 6 H), 1.15 (br, $10 \mathrm{H}$ ), 0.75 (br, $4 \mathrm{H})$ ppm; ${ }^{13} \mathrm{C}-\mathrm{NMR}\left(100 \mathrm{MHz}, \mathrm{CDCl}_{3}, \delta\right.$ ): 145.40, 141.93, 138.12, 133.52, 130.63, 128.87, 127,2, 121.6, 58.55, 43.54, 42.91, 41.50, 29.62, 29.40, 28.55, $25.11 \mathrm{ppm}$.

\section{Preparation of PFT@CB8 Complex}

PFT (10 mg, $0.02 \mathrm{mmol}$ ) was suspended in $10 \mathrm{~mL}$ of water and sonicated for $10 \mathrm{~min}$ and subsequently CB8 (40 mg,

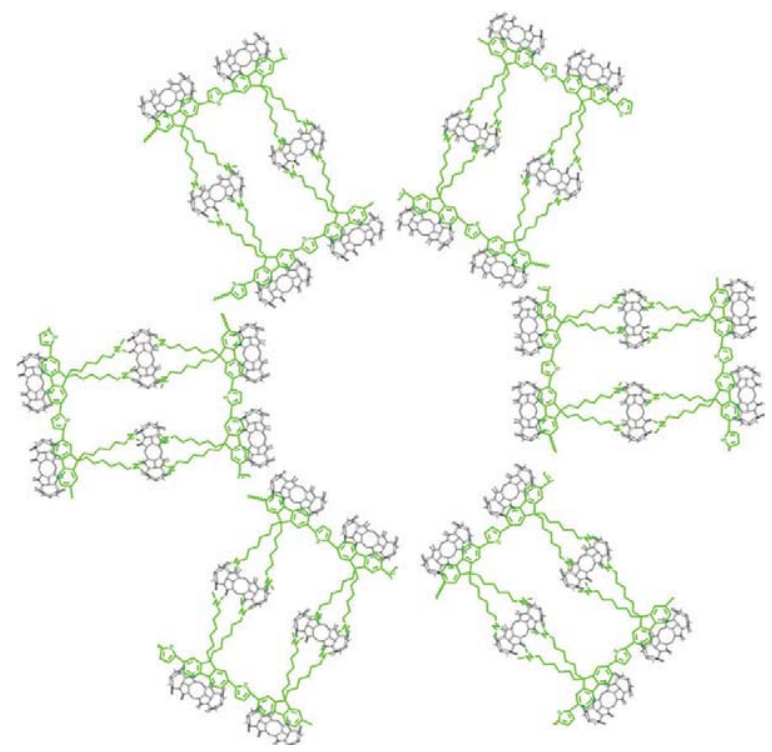

FIGURE 6 Suggested structure for the vesicles formation. [Color figure can be viewed in the online issue, which is available at wileyonlinelibrary.com.] 
$0.03 \mathrm{mmol}$ ) was added; the mixture was stirred overnight and undissolved particles were removed by suction filtration to obtain bright green emitting solution. The solvent was removed under reduced pressure to yield yellow powder.

\section{CONCLUSIONS}

The results point out that CB8 having a very low water solubility $(<150 \mu \mathrm{M})$ but with a large cavity $\left(\sim 300 \AA^{3}\right.$ capacity) encapsulates the part of the backbone of PFT and consequently through an inclusion complex formation the dissolution of nonionic PFT is facilitated. The structure of the complex was elucidated by NMR experiments including ${ }^{1} \mathrm{H}$ and selective 1D-NOESY. It was found out that there were strong NOEs between protons $H x, H y$ of CB8 and the protons of aromatic backbone. This complex emits green and is highly fluorescent with fluorescent quantum yield of 35\%. In contrast, CB6 or water-soluble CB7, although they are chemically identical to CB8 but having smaller cavity than CB8 do not have any effect on the dissolution and photophysical properties of PFT. It was also observed that the morphology of the polymer PFT was affected by the presence of CB8. Thus, CB8-assisted self-assembly of polymer chains causes vesicles formation; these structures were characterized by DLS, AFM, SEM, and TEM fluorescent optical microscopy. This finding offers a significant contribution to the area of water soluble-light emitting polymers. In particular, it will have great impact in the development of biological and chemical sensors owing to the solubility of nonionic conjugated polymers in water.

This work was partially supported by the Royal Society of Chemistry, T.W.J. Traveling Fellowship, Turkish Research Council (TUBITAK, 108T217) and EU FP7-UNAM-REGPOT Grant No: 203953. We are grateful to Oren Scherman for providing GPC and MALDI-MS facilities at Cambridge University.

\section{REFERENCES AND NOTES}

1 Burroughes, J. H.; Bradley, D. D. C.; Brown, A. R.; Marks, R. N.; Mackay, K.; Friend, R. H.; Burns, P. L.; Holmes, A. B. Nature 1990, 347, 539-541.

2 McQuade, D. T.; Pullen, A. E.; Swager, T. M. Chem Rev 2000, $100,2537-2574$

3 Pu, K.-Y.; Liu, B. Adv Funct Mater 2008, 18, 1321-1328.

4 Hoven, V.; Garcia, A.; Bazan, G. C.; Nguyen, T.-Q. Adv Mater 2008, 20, 3793-3810.

5 Liu, B.; Bazan, G. C. Chem Asian J 2007, 2, 499-504.

6 Huyal, O.; Ozel, T.; Tuncel, D.; Demir, H. V. Optics Express 2008, 16, 13391-13397.

7 Huyal, I. O.; Koldemir, U.; Ozel, T.; Demir, H. V.; Tuncel, D. J Mater Chem 2008, 18, 3568-3574.

8 Ho, H.-A.; Bèra-Abèrem, M.; Leclerc, M. Chem Eur J 2005, 11 , 1718-1724.

9 Scherf, U.; List, E. J. W. Adv Mater 2002, 14, 477-487.

10 Leclerc, M. J Polym Sci Part A: Polym Chem 2001, 39, 2867-2873.
11 Liu, B.; Wang, S.; Bazan, G. C.; Mikhailovsky, A. J Am Chem Soc 2003, 125, 13306-13307.

12 Brookins, R. N; Schanze, K. S.; Reynolds, J. R. Macromolecules 2007, 40, 3524-3526.

13 Lee, S.; Kim, J.-M. Macromolecules 2007, 40, 9201-9204.

14 Zhu, B.; Han, Y.; Sun, M.; Bo, Z. Macromolecules 2007, 40, 4494-4500.

15 Laurenti, M.; Rubio-Retama, J.; Garcia-Blanco, F.; Cabarcosm, E. L. Langmuir 2008, 24, 13321-13327.

16 Burrows, H. D.; Tapia, M. J.; Silva, C. L.; Pais, A. A. C. C.; Fonseca, S. M.; Pina, J.; Seixas de Melo, J.; Wang, Y.; Marques, E. F.; Knaapila, M.; Monkman, A. P.; Garamus, V. M.; Pradhan, S.; Scherf, U. J Phys Chem B 2007, 111, 4401-4410.

17 Frampton, M. J.; Anderson, H. L. Angew Chem Int Ed 2007, 46, 1028-1064.

18 Harada, A.; Hashidzume, A.; Yamaguchi, H.; Takashima, Y. Chem Rev 2009, 109, 5974-6023.

19 Grigoras, M.; Stafie, L. Supramol Chem 2010, 22, 237-248.

20 Kim, I.-S.; Jung, J.; Kim, S.-Y.; Lee, E.; Kang, J.-K.; Sakamoto, S.; Yamaguchi, K.; Kim, K. J Am Chem Soc 2000, 122, 540-541.

21 Kim, K.; Selvapalam, N.; Ko, Y. H.; Park, K. M.; Kim, D. Chem Soc Rev 2007, 36, 267-279.

22 Isaacs, L. Chem Commun 2009, 619-629.

23 Tuncel, D.; Tiftik, H. B.; Salih, B. J Mater Chem 2006, 16, 3291-3296.

24 Tuncel, D.; Ozsar, O.; Tiftik, H. B.; Salih, B. Chem Commun 2007, 1369-1371.

25 Tuncel, D.; Katterle, M. Chem A Eur J 2008, 14, 4110-4116.

26 Celtek, G.; Artar, M.; Scherman, O. A.; Tuncel, D. Chem A Eur J 2009, 15, 10360-10363.

27 Sindelar, V.; Silvi, S.; Parker, S. E.; Sobransingh, D.; Kaifer, A. E. Adv Funct Mater 2007, 17, 694-701.

28 Lee, J. W.; Hwang, I.; Jeon, W. S.; Ko, Y. H.; Sakamoto, S.; Yamaguchi, K.; Kim, K. Chem Asian J 2008, 3, 1277-1283.

29 Koner, A. L.; Nau, W. M. Supramol Chem 2007, 19, 55-66.

30 Wagner, B. D.; Stojanovic, N.; Day, A. I.; Blanch, R. J. J Phys Chem B 2003, 107, 10741-10746.

31 Wang, R.; Yuan, L.; Macartney, D. H. Chem Commun 2005, 5867-5869.

32 Bakirci, H.; Koner, A. L.; Nau, W. M. Chem Commun 2005, 43, 5411-5413.

33 Eelkema, R.; Maeda, K.; Odell, B.; Anderson, H. L. J Am Chem Soc 2007, 129, 12384-12385.

34 Ling, Y.; Kaifer, A. E. Chem Mater 2006, 18, 5944-5949.

35 Liu, Y.; Shi, J.; Chen, Y.; Ke, C.-F. Chem Int Ed 2008, 47, 7293-7296.

36 Jeon, Y. J.; Bharadwaj, P. K.; Cho, S. W.; Lee, J. W.; Kim, K. Angew Chem Int Ed 2002, 41, 4474-4476. 